\title{
Cross-field transport and pitch-angle anisotropy of solar energetic particles in MHD turbulence
}

\author{
F. Fraschetti \\ Departments of Planetary Sciences and Astronomy, University of Arizona, Arizona, USA \\ Correspondence to: F. Fraschetti (ffrasche@lpl.arizona.edu)
}

Received: 11 June 2015 - Revised: 2 October 2015 - Accepted: 30 November 2015 - Published: 15 January 2016

\begin{abstract}
Recent modelling of solar energetic particles (SEPs) propagation through the heliospheric turbulence, also discussed in this workshop, has investigated the role of the pitch-angle scattering and the perpendicular transport in spreading particles in heliolongitude, as shown by multi-spacecraft measurements (STEREO $\mathrm{A} / \mathrm{B}, \mathrm{ACE}, \mathrm{SOHO}$, etc.) at $1 \mathrm{AU}$ in various energy ranges. In some events the first-order pitch-angle anisotropy of the particles distribution is not-negligible. We calculate the average perpendicular displacement due to the gradient/curvature drift in an inhomogeneous turbulence accounting for pitch-angle dependence for two MHD turbulence models: (a) 3-D isotropic, (b) anisotropic as conjectured by Goldreich-Sridhar. We find in both cases that the drift scales as $\left(1-\mu^{2}\right)^{2}$ with the cosine of pitch-angle $\mu$, in contrast with previous models for transport of SEPs. This result can impact the models of propagation of SEPs through the heliosphere.
\end{abstract}

\section{Introduction}

In recent years, the multi-spacecraft monitoring by STEREO A/B, ACE, SOHO or Ulysses, has shown that SEP events spread in longitude more than $180^{\circ}$ at $1 \mathrm{AU}$ (Richardson et al., 2014) and also in latitude as far as $70^{\circ}$ (Malandraki et al., 2009). The interpretation of these measurements invokes two main agents: (1) extended or moving source in the interplanetary medium (CME-driven shocks); (2) strong perpendicular transport across the spiral magnetic field. Measurements of first-order anisotropy suggest that the pitch-angle dependence of the particle distribution is relevant to the interpretation of the longitudinal spread (Dröge et al., 2014). In situ measurements of longitudinal spread are particularly valuable in that they might probe whether the transport perpendicular to the average magnetic field is dominated by the meandering of the field lines or the transport away from the field lines; this issue has been recently investigated in Fraschetti and Jokipii (2011).

Test-particle simulations (Fraschetti and Giacalone, 2012) show that even in a weak three-dimensional isotropic turbulence charged particles decorrelate from the unperturbed magnetic field on a time scale comparable to the gyroperiod. In addition to repeated scattering in the pitch-angle by which particles move back and forth along a given field line, a prompt decorrelation reduces the initial anisotropy of the particles streaming from the source and enhances the angular spread. These results pinpoint to a propagation regime wherein early-time perpendicular transport cannot be neglected.

Recent phenomenological models of charged particle transport in the heliosphere include a dependence of the perpendicular diffusion coefficient on the pitch-angle of the particle velocity with respect to the global average magnetic field $(\mu)$ given by $\sqrt{1-\mu^{2}}$, suggested by a proportionality of the perpendicular diffusion coefficient to the particle gyroradius (Dröge et al., 2014). Different pitch-angle dependencies were considered in Strauss and Fichtner (2015).

In this short note we outline the calculation of the perpendicular transport coefficient due to gradient/curvature drift originating from the inhomogeneity of the magnetic turbulence for an anisotropic pitch-angle distribution of the particles. The results for the 3-D isotropic turbulence and the MHD anisotropic turbulence conjectured by Goldreich and Sridhar (1995), hereafter GS95, are compared. In Fraschetti and Jokipii (2011) we have calculated the average over an isotropic pitch-angle distribution; in this short note we relax such an assumption. We do not calculate here the contribu- 
tion to the perpendicular transport due to field line meandering.

\section{Gradient/curvature drift transport}

\subsection{Three-dimensional isotropic}

We consider a spatially homogeneous time-independent magnetic field with superimposed fluctuations. The amplitude of the fluctuation $(\delta B)$ is assumed to be much smaller than the average field magnitude $\left(B_{0}\right)$. We represent such a magnetic field as $\mathbf{B}(\mathbf{x})=\mathbf{B}_{0}+\delta \mathbf{B}(\mathbf{x})$, with an average component $\mathbf{B}_{0}=B_{0} \mathbf{e}_{z}$; we assume that the average over field realisations vanishes, $\langle\delta \mathbf{B}(\mathbf{x})\rangle=0$, and $\delta B(\mathbf{x}) / B_{0} \ll 1$. We make use of the gyroperiod averaged guiding-center velocity transverse to the field $\mathbf{B}(\mathbf{x})$, i.e., $\mathbf{V}_{\perp}^{G}(t)$, to the first order in $\delta B(\mathbf{x}) / B_{0}$, given for a particle of speed $v$, momentum $p$ and charge $Z e$ by

$\mathbf{V}_{\perp}^{G}(t)=\frac{v p c}{Z e B}\left[\frac{1-\mu^{2}}{2} \frac{\mathbf{B} \times \nabla B}{B^{2}}+\mu^{2} \frac{\mathbf{B} \times(\mathbf{B} \cdot \nabla) \mathbf{B})}{B^{3}}\right]$

where $\mu$ is the cosine of the pitch-angle with respect to the average field $B_{0}$ (Rossi and Olbert, 1970; Fraschetti and Jokipii, 2011). The average square transverse displacement of the particle guiding center from the direction of local $B$ due to drift along the axis $x_{i}, d_{D_{i i}}(t)$, at time $t$ is written as

$d_{D_{i i}}(t)=\int_{0}^{t} d \xi\left\langle\mathbf{V}_{\perp, i}^{G}\left(t^{\prime}\right) \mathbf{V}_{\perp, i}^{G}\left(t^{\prime}+\xi\right)\right\rangle$.

In the Eq. (1) we make use of the Fourier representation for $\delta \mathbf{B}(\mathbf{x})$ and assume an inertial range magnetic turbulence power spectrum which is uncorrelated at different wavenumber vectors: $\left\langle\delta B_{r}(\mathbf{k}) \delta B_{q}^{*}\left(\mathbf{k}^{\prime}\right)\right\rangle=\delta\left(\mathbf{k}-\mathbf{k}^{\prime}\right) P_{r q}(\mathbf{k})$. As in Fraschetti and Jokipii (2011) we use

$$
\begin{aligned}
& P_{r q}(\mathbf{k})=\frac{G(k)}{8 \pi k^{2}}\left[\delta_{l m}-\frac{k_{l} k_{m}}{k^{2}}\right], \\
& \text { with } \quad G(k)=\left\{\begin{array}{cc}
G_{0} k^{-q} & \text { if } k_{\min }<k<k_{\max } \\
G_{0} k_{\min }^{-q} & \text { if } k_{0}<k<k_{\min },
\end{array}\right.
\end{aligned}
$$

where $k^{\max }$ corresponds to the scale where the dissipation rates of the turbulence overcomes the energy cascade rate, the coherence length is given by $L=2 \pi / k^{\mathrm{min}}$, and the physical scale of the system by $2 \pi / k^{0}$.

With these assumptions, the cross-field diffusion coefficient (limit for large times of the average square displacement) associated to gradient/curvature drift of the guiding center due to the turbulence inhomogeneity (in other words the diffusive motion of the guiding center away from the field lines) reads

$D_{D}^{i}(t) \rightarrow \frac{\pi}{16}\left(\frac{\delta B}{B_{0}}\right)^{2}\left|\frac{q-1}{q-2}\right| \frac{2 \pi v^{3}}{L \Omega^{2}}\left(\frac{1-\mu^{2}}{2}\right)^{2}$
We note that $1 / 2 \int_{-1}^{1} d \mu D_{D}^{i}(t)=d_{D}^{i}(t)$, where $d_{D}^{i}(t)$ is the time-dependent average square displacement in Eq. (45) of Fraschetti and Jokipii (2011). The condition that the perpendicular transport time-scale is much shorter than the parallel one is translated into the restriction of Eq. (4) to times $t<1 / k_{\min } v_{\|} \simeq L / 2 \pi v_{\|}$; thus, for higher energy particles the physical time this regime applies is shorter because the perpendicular scale grows with the particle gyroradius $r_{\mathrm{g}}$ and the perpendicular transport becomes relevant. For a $25 \mathrm{MeV}$ proton (within the energy range of STEREOs and SOHO instruments) at $1 \mathrm{AU},\left(\delta B / B_{0}\right)^{2}=0.1, r_{\mathrm{g}} / L \simeq 0.1(L=0.01 \mathrm{AU})$ and $q=11 / 3$, we have $D_{D}^{i}(t) \simeq 4.5 \times 10^{17}\left(1-\mu^{2}\right)^{2} \mathrm{~cm}^{2} \mathrm{~s}^{-1}$ for $t<1 / k_{\min } v_{\|} \simeq 3.5 \mathrm{~s}$.

\subsection{MHD anisotropic turbulence GS95}

Also in this case we consider a spatially homogeneous, fluctuating, time-independent global magnetic field $\mathbf{B}_{0}$ and decompose the field as $\mathbf{B}(\mathbf{x})=\mathbf{B}_{0}+\delta \mathbf{B}(\mathbf{x})$, with a large-scale average component $\mathbf{B}_{0}=B_{0} \mathbf{e}_{z}$ and a fluctuation $\langle\delta \mathbf{B}(\mathbf{x})\rangle=0$. We assume that the inertial range extends from $L$ down to some scale wherein injected energy ultimately must dissipate. We introduce a scale $\hat{L}$ such that $r_{\mathrm{g}} \ll \hat{L} \ll L$. The fluctuations are large compared to the total magnetic field at scale $\sim L$; however, at scales $<\hat{L}$ the fluctuations are small compared to the local average field, and the first-order orbit theory applies to eddies up to scale $\hat{L}$. By using similar assumptions, Chandran (2000) made use of the quasi-linear theory to calculate parallel transport coefficient in GS95.

According to the GS95 conjecture, the pseudo-Alfvén modes are carried passively by the shear-Alfvén modes with no contribution to the turbulence cascade to small scales which is seeded by collisions of shear modes only. Fraschetti (2015) shows that the transport perpendicular to the local average field due to gradient/curvature drift is dominated by the power of the pseudo-Alfvén modes along the local average field given by

$$
\begin{aligned}
& P_{33}\left(\hat{k}_{\|}, \hat{k}_{\perp}\right)=\frac{\hat{k}_{\perp}^{2}}{\hat{k}^{2}} \Pi\left(\hat{k}_{\|}, \hat{k}_{\perp}\right), \\
& \quad \text { with } \Pi\left(\hat{k}_{\|}, \hat{k}_{\perp}\right)=\frac{\varepsilon \mathcal{N} B_{0}^{2}}{\ell^{1 / 3}} \hat{k}_{\perp}^{-10 / 3} \exp \left(-\frac{\ell^{1 / 3} \hat{k}_{\|}}{\hat{k}_{\perp}^{2 / 3}}\right) .
\end{aligned}
$$

where $\hat{k}_{\|}, \hat{k}_{\perp}$ indicate wave numbers parallel and perpendicular, respectively, to the local average field, $\ell$ is the outer scale of the pseudo-modes, $\varepsilon$ is the power in the pseudorelative to shear-modes $(0<\varepsilon<1)$ and $\mathcal{N}$ is a normalisation constant accounting for both polarisation modes given by $\mathcal{N} \simeq \frac{1}{3 \pi}\left(\delta B / B_{0}\right)^{2}(1+\varepsilon(L / \ell))^{-1}$, that includes also scales between $\hat{L}$ and $L$, as it can be easily seen that the contribution to the turbulent power from perpendicular scales $\hat{L}<k_{\perp}^{-1}<L$ is exponentially suppressed.

Following the calculation in the Appendix of Fraschetti (2015), we find that the average square displacement due to 
gradient/curvature drift is given by

$$
\begin{gathered}
D_{D}^{a}(t) \simeq \frac{1}{8}\left(\frac{\delta B}{B_{0}}\right)^{2} \frac{\varepsilon}{1+\varepsilon L / \ell}\left(\frac{L}{\ell}\right)^{1 / 3} \\
\frac{v^{4}}{\Omega^{3} L^{2}} \Omega t\left(k_{\perp}^{M} L\right)^{2.2}\left(\frac{1-\mu^{2}}{2}\right)^{2} .
\end{gathered}
$$

Due to the lack of space, we omit here the corresponding result for the shear- modes. For a $25 \mathrm{MeV}$ proton at $1 \mathrm{AU}$, by taking $L=l, \varepsilon=0.5,\left(\delta B / B_{0}\right)^{2}=0.1$ and $\left(k_{\perp}^{M}\right)^{-1} \simeq$ $10^{-5} \mathrm{AU}$, we have $D_{D}^{a}(t) \simeq 2.5 \times 10^{21}\left(1-\mu^{2}\right)^{2} t[\mathrm{~s}] \mathrm{cm}^{2} \mathrm{~s}^{-1}$ for $t<1 / k_{\|}^{\min } v_{\|} \simeq 3.5 \mathrm{~s}$. We emphasise that such a drift cumulates for hours, that is the typical time-scale of the longitudinal spread of SEP, resulting in a possibly significant contribution.

\section{Conclusions}

We have calculated the time-dependent average square displacement $\mathrm{DD}(\mathrm{t})$ due to gradient/curvature drift for two distinct MHD turbulence models: (a) 3-D-isotropic and (b) anisotropic as conjectured by Goldreich and Sridhar (1995); the assumption of the pitch-angle isotropy of the particle distribution function has been relaxed to account for recently measured first order anisotropy. In both cases, we find $D_{D} \propto\left(1-\mu^{2}\right)^{2}$; such a dependence arises from the gradient drift that is proportional to the particle kinetic energy normal to the field $\left(p_{\perp}^{2} / 2 m\right.$ for a particle of mass $\left.m\right)$. We conclude that spacecraft data compatible with the scaling $\left(1-\mu^{2}\right)^{2}$ would support at once the Goldreich-Sridhar conjecture and our model for perpendicular transport. Although drifts cannot be neglected in the interpretation of multi-spacecraft SEP data across the Parker spiral (Marsh et al., 2013), the meandering of the field lines is expected to be larger than drifts in perpendicular transport at scales close to the outer scale. This effect will be assessed in a separate work.

Acknowledgements. The author acknowledges useful discussions with W. Dröge and R. D. Strauss and constructive feedback of the referees. This work was supported, in part, by NASA under grant NNX13AG10G. This work benefited from discussions at the team meetings "First principles physics for charged particle transport in strong space and astrophysical magnetic turbulence" at ISSI in Bern, Switzerland.

Edited by: P. Desiati

Reviewed by: two anonymous referees

\section{References}

Chandran, B. D. G.: Scattering of Energetic Particles by Anisotropic Magnetohydrodynamic Turbulence with a Goldreich-Sridhar Power Spectrum, Phys. Rev. Lett., 85, 4656-4659, 2000.

Dröge, W., Kartavykh, Y. Y., Dresing, N., Heber, B., and Klassen, A.: Wide longitudinal distribution of interplanetary electrons following the 7 February 2010 solar event: Observations and transport modeling, J. Geophys. Res., 119, 6074-6094, 2014.

Fraschetti, F.: Cross-field transport in Goldreich-Sridhar MHD turbulence 2015, arXiv/1512.05352, online first, 2015.

Fraschetti, F. and Giacalone, J.: Early-time velocity auto-correlation for charged particles diffusion and drift in static magnetic turbulence, Astrophys. J., 755, 114, 9 pp., 2012.

Fraschetti, F. and Jokipii, J. R.: Time-dependent perpendicular transport of fast charged particles in a turbulent magnetic field, Astrophys. J., 734, 83, 8 pp., 2011.

Goldreich, P. and Sridhar, S.: Toward a theory of interstellar turbulence. 2: Strong alfvenic turbulence, Astrophys. J., 438, 763-775, 1995.

Malandraki, O. E., Marsden, R. G., Lario, D., Tranquille, C., Heber, B., Mewaldt, R. A., Cohen, C. M. S., Lanzerotti, L. J., Forsyth, R. J., Elliott, H. A., Vogiatzis, I. I., and Geranios, A.: Energetic Particle Observations and Propagation in the Three-dimensional Heliosphere During the 2006 December Events, Astrophys. J., 704, 469-476, 2009.

Marsh, M. S., Dalla, S., Kelly, J., and Laitinen, T.: Drift-induced Perpendicular Transport of Solar Energetic Particles, Astrophys. J., 774, 4, 9 pp., 2013.

Richardson, I. G., von Rosenvinge, T. T., Cane, H. V., Christian, E. R., Cohen, C. M. S., Labrador, A. W., Leske, R. A., Mewaldt, R. A., Wiedenbeck, M. E., and Stone, E. C.: $>25 \mathrm{MeV}$ Proton Events Observed by the High Energy Telescopes on the STEREO A and B Spacecraft and/or at Earth During the First Seven Years of the STEREO Mission, Sol. Phys., 289, 3059-3107, 2014.

Rossi, B. and Olbert, S.: Introduction to the Physics of Space, McGraw-Hill, New York, 1970.

Strauss, R. D. and Fichtner, H.: On aspects pertaining to the perpendicular diffusion of solar energetic particles, Astrophys. J., 801, 29, 2015. 